Provided by the author(s) and University of Galway in accordance with publisher policies. Please cite the published version when available.

\begin{tabular}{|c|l|}
\hline Title & Fast A/V channel switching on a wireless home network \\
\hline Author(s) & Corcoran, Peter; Callaly, Frank \\
\hline $\begin{array}{c}\text { Publication } \\
\text { Date }\end{array}$ & 2005-11-21 \\
\hline $\begin{array}{c}\text { Publication } \\
\text { Information }\end{array}$ & $\begin{array}{l}\text { Channel Switching on a Wireless Home Network. Paper } \\
\text { presented at the Computer as a Tool, 2005. EUROCON } \\
\text { 2005.The International Conference on. }\end{array}$ \\
\hline $\begin{array}{c}\text { Publisher } \\
\text { IEEE }\end{array}$ \\
\hline $\begin{array}{c}\text { Link to } \\
\text { publisher's } \\
\text { version }\end{array}$ & http://dx.doi.org/10.1109/EURCON.2005.1630201 \\
\hline $\begin{array}{c}\text { Item record } \\
\text { http://hdl.handle.net/10379/1612 }\end{array}$ \\
\hline
\end{tabular}

Downloaded 2023-04-26T11:28:03Z

Some rights reserved. For more information, please see the item record link above. 


\title{
Fast A/V Channel Switching on a Wireless Home Network
}

\author{
Peter Corcoran, member IEEE and Frank Callaly
}

Abstract - A method of enabling fast switching between multimedia streams on an $802.11 \mathrm{~g}$ network is described. This technique is particularly useful for $\mathrm{A} / \mathrm{V}$ home network applications where it is desirable to simulate the fast channel switching of conventional analog TV channels in order to emulate the behaviour of multichannel TV. ${ }^{I}$

Index Terms - Multimedia, Video \& Audio Streaming, Home Networks, and WiFi (802.11).

\section{[1] INTRODUCTION}

In current practice, broadcast media (TV, radio) are distributed to receivers that have essentially no latency. From the users perspective no perceptible time elapses from the moment that a media channel is selected to the time that audiovisual components of the media are seen and heard by the user. Consumers have become accustomed to this characteristic and often use it to scan through available sources of content by "channel flicking".

Media streaming over a local area network, or on the Internet currently uses receivers that have significant latency. A perceptible amount of time elapses from the moment that content is selected to the time that it begins to play. This occurs because the TCP/P protocol does not carry messages with guaranteed quality of service, but rather attempts to deliver messages on a "best effort" bas is. Since messages may be delayed or even dropped and retried, media receivers buffer several messages of the media stream before playing any audio or video. This buffering delay can amount to as much as half a minute.

As a consequence the experience of media stream selection on a network is quite different from that of broadcast media, requiring more care on the part of the end user. Navigation methods for selecting a media stream may give static or pseudo-static images from the stream, together with descriptive information about the stream (e.g., album labels). All of these are designed to give the end user more information before he or she selects the stream, waits during the buffering delay, and finally evaluates the stream by listening to it and watching it, if possible.

A user may reject the stream almost immediately for many reasons, those including the sound or picture quality or the relevance of the stream to his or her interests. Unfortunately conventional $\mathrm{A} / \mathrm{V}$ applications are not able to describe the stream in sufficient detail so that the stream can be adequately evaluated by an end user without the disadvantages of buffer latency.

\section{[2] The. Emerging Home A/V Network Infrastructure}

Traditionally the home has been filled with stand-alone CE appliances such as the TV set or single add-on appliances such as a VCR or DVD player which allow users to record TV programmes and to play pre-recorded movies.

\footnotetext{
${ }^{1}$ This work is supported under the CFTD Frogram (CFTD/003/226) of Enterprise Ireland under the National Development Plon.

Peter Corcoran is with the Dept. Electronic Engineering, National University of Ireland, Galway (e-mail: peter.corcoran@inugal way,ie)

Frank Callaly is with the Consmer Electronics Research Group in the Dept. of Electronic Engineering National University of Ireland, Galway (email: franko@wumuz.nuigalway.ie )
}

Recently, next generation digital appliances have emerged, for example, PVRs (personal video recorders such as TiVo, Sky+, etc) and media adapters. A media adapter is an appliance which can receive streamed digital video or music over a network connection and convert it to standard RCA or S-Video output for presentation on a standard TV set. The PrismIQ ${ }^{\text {TMI }}$ is a good example of such an appliance [1].

Also, 802.11 wireless LAN (WLAN) technology is emerging as a means of home $A / V$ networking. The cost of $802.11 \mathrm{~g}$ access points has fallen below 100 Euro which is beginning to drive the market for networked CE products as consumers begin to perceive the benefits and simplicity of these new wireless networking technologies. However WLAN technology is more restricted in its bandwidth than conventional wired network connections and, being a wireless technology, it is more susceptible to packet loss and other inconsistent behavioural aspects.

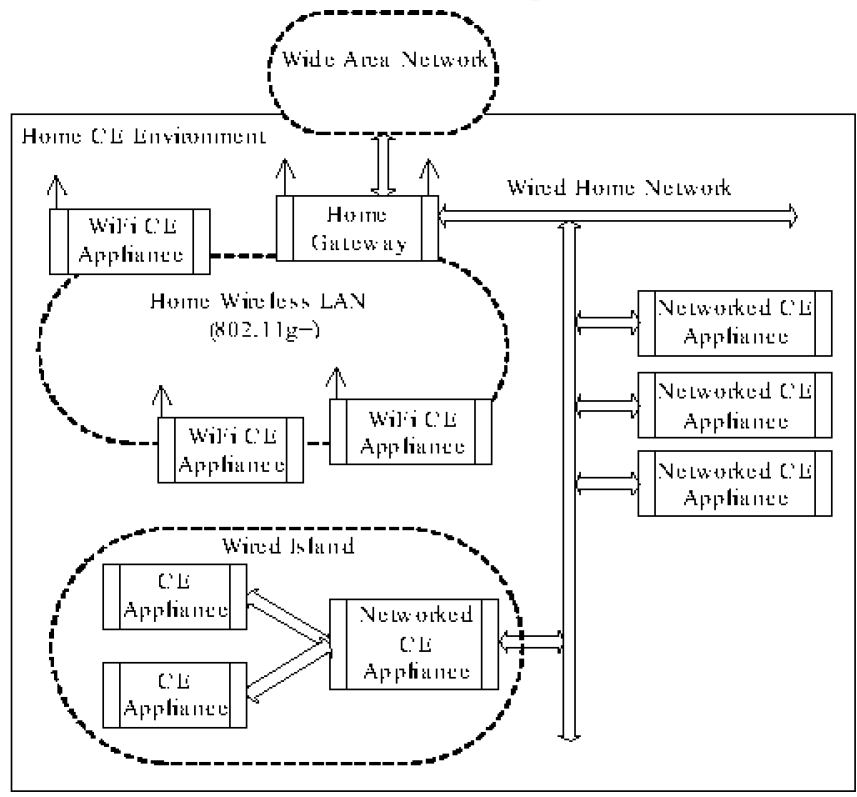

Fig 1: The emerging Home $A / W$ Network

Fig 1 : shows a home networking environment including next-generation wireless CE appliances, a home gateway appliance, more conventional wired $\mathrm{CE}$ appliances which can include personal computers, and a legacy wired island which contains legacy point-to-point CE appliances such as DVD players, Home Theater units, TV sets, etc. Such a wired island can be network enabled using a specialized network appliance such as a media adapter which was previously described. This home network is typically connected via the gateway appliance to an external wide area network (WAN), effectively forming the broadband connection to the home.

We note that it is quite practical to construct such a network with today's technology and we have built just such a wireless $\mathrm{A} /$ network in our laboratory. Desktop PCs offer a convenient and inexpensive platform for prototyping and are organized into clusters of 4 . Each PC is WiFi enabled and can be configured to behave as a variety of different $\mathrm{A} / \mathrm{V}$ appliances using a variety of networking protocols. The networking infrastructure used in our 
laboratory is described in a first companion paper [2] and the creation of a variety of networked $\mathrm{A} / \mathrm{V}$ appliances and the integration of these appliances into the home network is described in a second companion paper [3].

Next generation home networks will be based on TCP/P networking technology. However there are additional layers of software infrastructure present in a home network. These additional software layers are required to support "configuration-free" networking of devices and to facilitate and support device interoperability. When CE devices are connected to a home network they typically obtain an IP address automatically - this service is well known in desktop computer networking and is typically provided by a DHCP server. However a CE device must also advertise its capabilities to other devices on the network, obtain information on existing network devices \& services which are relevant to its functionality and it must also provide support for the user interface (UT) mechanisms supported by the home network. These additional software layers are collectively known as home networking middleware.

Probably the best known home networking middleware is Universal Plug \& Play (UPnP) technology which is incorporated in the latest versions of the Microsoft Windows OS [4]. Other well known middleware includes the Java-based JNI technology [5], the HAVi home gateway infrastructure supported by an industry consortium [6] and Zeroconf [7].

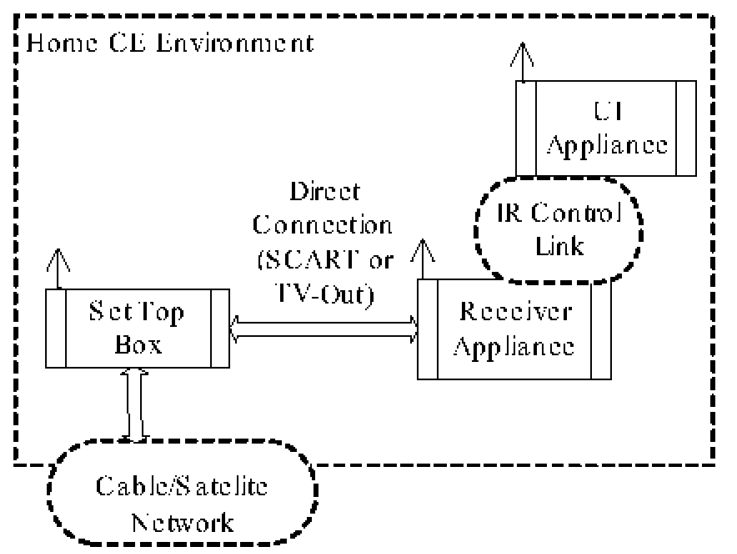

Fig 2(a): Prior Ant Analog Video Infrastructure

Fig 2(a) shows a prior art multi-channel AVV entertainment system. Such a system will access multiple AN channels over a cable or satellite network via a set-top box. A direct wired $\mathrm{A} / \mathrm{V}$ connection such as a SCART or TV-out provides analog $\mathrm{A} / \mathrm{V}$ signals to a receiver appliance which can be a standard TV set. Control of $\mathrm{A} / \mathrm{V}$ channel switching is achieved over an IR link from a UI appliance, which will typically be an InfraRed remote control with conventional keypad.

Fig 2(b) illustrates a home network configuration which can take advantage of the multimedia channel switching system described in this paper. Multimedia streams are generated by a local multichannel video stream server, which may access multimedia content either via a cable/satellite network, from a hard disk within the multichannel video server, or from extemal servers on a wide area network.

The multichannel server, user interface and receiver appliances coexist on a shared home network. When a new appliance joins such a network, information about its services, capabilities and user interface (UI) components are broadcast on the network. In a simple peer to peer home network architecture other appliances must remember this information about the device if they wish to access it later. In a system with a dedicated middleware server this information is stored on a central network server/gateway device.

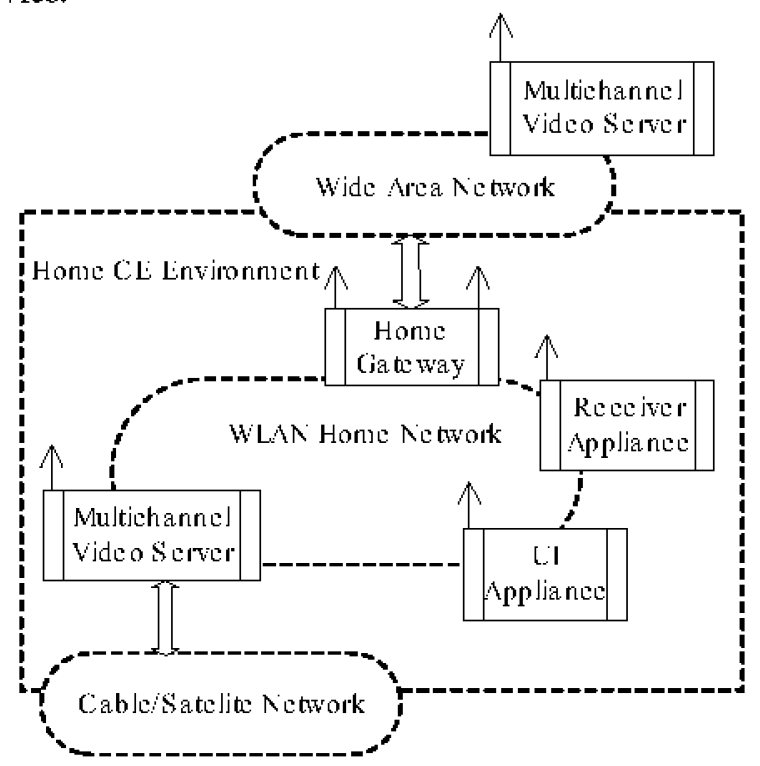

Fig 2(b): Video Infrastracture for WiFi Home Network

\section{[3] Stream Switching Archtecture}

In normal viewing mode the action of switching a streamed video channel using the infrastructure illustrated in Fig 2(b) requires that a new multimedia stream connection be established and the previous connection be correctly terminated. This process will typically cause a delay of at least several seconds every time a channel switch occurs. The goal of our stream switching architecture is to allow a user to switch quickly between media streams without a need to wait for stream buffers to fill on each channel switch. It is clear that if we buffered every media stream at the receiver appliance this would eliminate the latency associated with such stream switching but the network is unlikely to be able to meet the bandwidth requirements of such a solution.

For example, a DVD-quality video stream transmitted in MPEG-2 format has a bandwidth requirement in the order of 8 Mbps. A typical $802.11 \mathrm{~g}$ network operating in standard mode has a practical throughput of $20 \mathrm{Mbps}$ which cannot support more than two concurrent MPEG-2 streams. The situation is improved with MPEG-4 compression where DVD quality can typically be achieved with a peak bandwidth requirement of the order of $2 \mathrm{Mbps}$. Thus an $802.11 \mathrm{~g}$ network can support up to 10 concurrent video streams.

Our approach is to buffer a limited number of neighbouring streams at a lower quality than the main viewing stream. If we consider a typical channel-switching application where a video server delivers multiple streams derived, say, from a cable TV network then we can refer to the channel which is currently being viewed as channel $\mathrm{N}$. As the standard behaviour of a user is to switch up or down by one channel at a time we choose to buffer channels $\mathrm{N}+2$, $\mathrm{N}+1$, and $\mathrm{N}-1, \mathrm{~N}-2$ at a lower quality, which results in a lower bitrate. 
Fig 3 illustrates the functionality of the start-up phase of the fast channel switching mode. The main buffered stream has been previously selected as the current channel $\mathrm{N}$, and as it will normally be in an encoded format such as MPEG2 or MPEG4 it must next be decoded and converted to a format suitable for display.

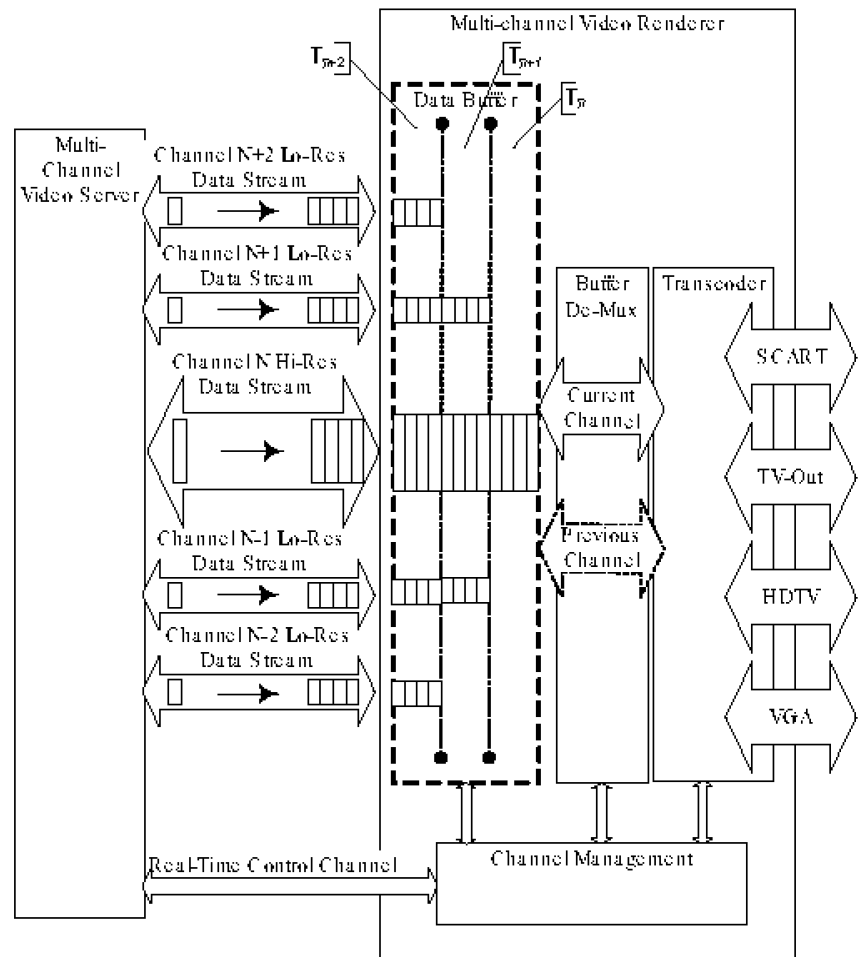

Fig 3: Video Switching Architecture in Start-Up Mode

Note that at the time the main steam has buffered sufficient data to activate the video rendering subsystems the buffers for the surrounding low quality channels are only beginning to fill. This is because the client takes a fixed amount of time to establish each of these new stream connections. The actual number of additional low quality channels to be streamed, and the bitrate of these streams is determined by the channel management subsystem as will be described shortly. We also remark that after the system has remained in steady-state for a predetermined time interval, i.e. the user has settled into viewing a single channel, then the channel management module can be configured to deactivate these additional low-quality streams.

Fig 4 illustrates the operation of our architecture during a channel switch. It is assumed that the multi-channel video server is selected at a particular channel, $\mathrm{N}$, and that four additional low quality video channels, $\mathrm{N}-2, \mathrm{~N}-1, \mathrm{~N}+\mathrm{1}$ and $\mathrm{N}+2$ are streamed at the same time. Immediately after a user switches channels the new main channel will initially be available at a lower quality. However, if the user continues to view the same channel its quality will begin to improve after a short time, typically equal to the buffer latency.

The reduction in quality is achieved using one of two methods. The first technique is to reduce the spacial resolution of the video stream, e.g. if the main stream is a video using VGA resolution, then the low quality streams can use QVGA thus significantly reducing the bandwidth required by the stream.

In practical use the difference between viewing content at VGA or QVGA resolution is negligible when deciding if content is worthwhile viewing. If the rendering device can support line-doubling then QVGA quality is very acceptable on a conventional TV-set. For higher resolution screens such as Plasma-TV it is preferable to employ SVGA resolution (DVD-quality) for the main video stream while QVGA is still adequate for the low-resolution channels, although there is usually sufficient bandwidth to support VGA resolution unless it is desired to operate several multi-channel clients with full DVD-video quality.

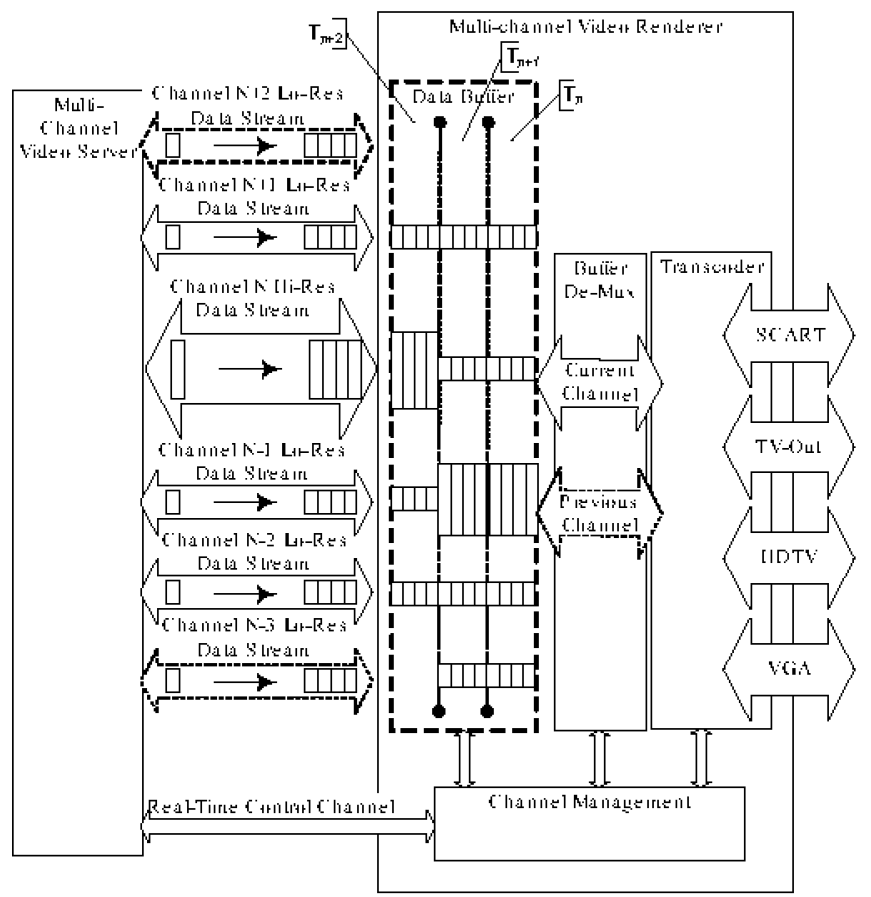

Fig 4: Architecture Behaviour during Channel Switch.

The second quality reduction technique, referred to as requantisation, is well known in video processing [8]. This technique uses the characteristics of DCT based encoders (e.g. MPEG2) to reduce the number of bits in a video stream. Quantisation is the process whereby the amount of information contained in a block of DCT coefficients is reduced, at the expense image quality. DVD quality MPEG2 video has a low quantisation factor, and so the video quality is very high. By increasing the quantisation factor when a stream is encoded the number of bits used to encode each DCT coefficient is reduced, and so the overall bitrate of the stream is reduced. The advantage of this technique is that it can be applied to a stream without it being fully decoded and re-encoded. Thus the processing requirements of the Multi-Channel Video Server are reduced.

The scaling techniques associated with the MPEG2/4 standards [9][10] were considered as alternatives to those chosen. It was decided not to use them as the methods chosen can be applied quite easily to almost any video coding format, and they do not require the decoder to support any special scaling profile. However, this architecture does not prevent the use of specialized scalable encoding in future revis ions.

\section{[3] SYSTEM WORKFLOW}

Fig 5 is a flow chart illustrating the principle functional steps of fast channel switching mode. An initialization phase optionally involves a determination of the number of peripheral multimedia streams and/or the minimum bitrate 
of a stream. If this step is skipped then predetermined values are used for these settings.

Acquiring the peripheral streams is next initiated and once the system buffer threshold level is achieved the main fast switching mode is enabled. In the main functional loop the system first determines if a user has requested a channel switch. If this is so then a fast channel switch is initiated. If a channel switch has not been requested the system optionally tests if a timer has expired for increasing the quality of main channel. Using the technique of requantising a stream, described in Section 3, dynamic adjustments can be made to the quality of a stream. Thus, in certain configurations the system can incrementally improve the quality of the viewed channel every few seconds as it is watched over a period of, say, a few tens of seconds.

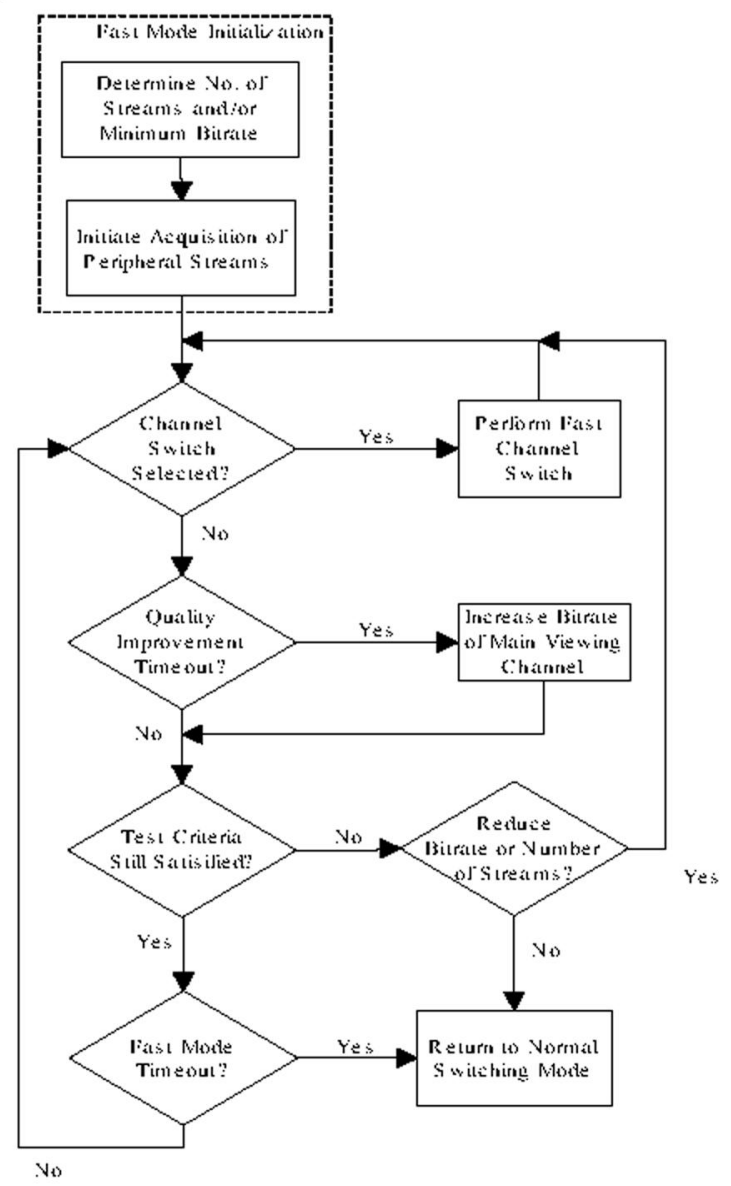

Fig 5: Main Workflow to Control System Modes

The next step is to determine if one or more test criteria, typically associated with conditions on the network, are still valid. For example, the system may not initiate fast mode unless a certain minimum bandwidth, $B$, is available on the home network but it may be acceptable to remain in fast mode even if the available bandwidth drops as low, say, $75 \%$ of $\mathrm{B}$. If the test criteria are no longer satis fied then the system switches back to normal (single channel) mode. However an optional step first allows it to attempt to reduce the bitrate or number of streams.

\section{[4] Conclusions}

In general, media stream receiving applications will buffer approx. 3-5 seconds of video [11], and when a live TV stream is being received the server cannot send the video any faster than it is receiving it. This means that the delay experienced by the user is equal to the amount of video that the application buffers, plus any additional time taken by the software to initialize playback. This extra time should be very small relative to the buffering time.

Using the system described in this paper, we have been able to reduce channel switching times to just that required by the software to begin playback. In measurements we were able to reduce this switching time to as little as 40 $70 \mathrm{~ms}$.

Our experimental receiver consisted of a Desktop PC with a Pentium 4 processor, Linux kemel version 2.6.11, using MPEG-2 and MPEG-4 video. The software is implemented in $\mathrm{C}$, so as to be as efficient as possible. The switching time has been found to vary due to factors such as the type of video encoding used for the stream and the number of decoded frames that are buffered by the display engine.

It is clear that this system can greatly reduce the time required to switch between $\mathrm{A} / \mathrm{V}$ streams and can make the experience of viewing live video streams over a network more enjoyable.

\section{[5]ACKNOWLEDGMENT}

This research was funded by Enterprise-Ireland's Commercialization Fund, Project CFTD/03/226.

\section{[7]REFERENCES}

[1] PrismIQ ${ }^{\mathrm{TM}}$ mediaplayer technical specifications, October 2004, http://www.prismiq.com/support/player_techspecs.asp

[2] "Home Networking Middleware Infrastructure for Improved Audio/Video Appliance Functionality and Interoperability", Corcoran P., Callally F, and Cucos, A., Eurocon 2005.

[3] "Rapid Prototyping of Networked AV CE Appliances" Corcoran, P and Callally F., Eurocon 2005.

[4] "Understonding Universal Plug and Ploy", Microsoft Corp., http:/www.upnp .org/resources/whitepapers.asp

[5] "Jini Network Technology - Executive Overview", Sun Microsystems Inc. 200\%, http://www.sun.com/software/jini/whitep apers/jiniexecoverview.pdf

[6] "HAVi, the AV digital network revolution", HAVi organization http:/www.havi.org/techinfo/docshavi.pdf [

[7] "Autoconfiguration for IP Networking: Enobling Loca Communication", Erik Gutman June 2001, IEEE Intemet Computing

[8] "Video Compression Demystified", Symes Peter D, Dec 2000 London: McGraw-Hill

[9] 'Generic coding of moving pictures and associated audio (MPEG-2), ISO/LEC 13818

[10] "Overview of Fine Granularity Scalabitity in MPEG-4 Video Standard", Li Weiping March 2001, IEEE Transactions on Circuits and Systems for Video Technology, Vol 11, No 3 .

[11] "Reducing Broadcast Delay", Bill Birney, Microsoft Corp, April 2001 http:/www.microsoft.com/windows/windowsmedia/howto/articles/Br oadcastDelay.aspx

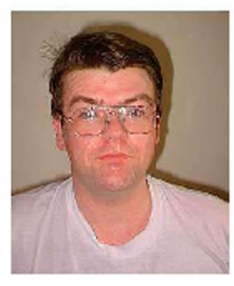

Peter Corcoran received the BAI (Electronio Engineering], BA (Math's) and a Ph.D. in the Theory of Dielectric Liquids from Trinity College Dublin in 1984 and 1986 . He was subsequently appointed to a lectureship in Electronic Engineering at NUL Galway. $\mathrm{He}$ is also director of IP for FotoNation Ireland Ltd. His current research interests include embedded systems, home networking and multimedia and wireless network technol ogies.

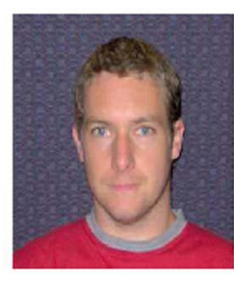

Frank Callaly received the B.Eng, (Electronic \& Computer Engineering) degree from the Nationa University of Irel and, Galway in 2003. He continued his studies with the Consumer Electronics Research Group at the National University of Ireland, Galway and is currently pursuing a Ph.D. degree in Electronio Engineering. His current research interests include digital video encoding, multimedia delivery techniques and home networking. 